\title{
Formación de la capacidad de los estudiantes para la actividad cognitiva en el proceso de aprendizaje
}

\section{Formation of students' ability to cognitive activity in the learning process}

\author{
Elena M. Chertakova \\ Togliatti State University, Togliatti, Russia. \\ ORCID: https://orcid.org/0000-0002-2923-5669
}

Anna V. Lapshova

Minin Nizhny Novgorod State Pedagogical University, Nizhny Novgorod, Russia. ORCID: https://orcid.org/0000-0001-7017-3589

\section{Olga I. Vaganova}

Minin Nizhny Novgorod State Pedagogical University, Nizhny Novgorod, Russia. ORCID: https://orcid.org/0000-0001-8347-484X

\section{Marina N. Bulaeva}

Minin Nizhny Novgorod State Pedagogical University, Nizhny Novgorod, Russia. ORCID: https://orcid.org/0000-0002-9928-9451

\section{Natalia V. Bystrova}

Minin Nizhny Novgorod State Pedagogical University, Nizhny Novgorod, Russia. ORCID: https://orcid.org/0000-0002-4310-6142

*Correspondence

Email: ivannovadremova29@gmail.com

\section{Cite as:}

Chertakova, E.M., Lapshova, A.V., Vaganova, O.I., Bulaeva M.N., Bystrova, N.V. (2021). Formación de la capacidad de los estudiantes para la actividad cognitiva en el proceso de aprendizaje. Propósitos y Representaciones, 9 (SPE1), e919. Doi: http://dx.doi.org/10.20511/pyr2021.v9nSPE1.919 


\section{Resumen}

Las profundas transformaciones que se han producido en el ámbito de la formación profesional de los estudiantes se han convertido en una respuesta a los cambios socioeconómicos del país. Los procesos innovadores tienen como objetivo satisfacer las necesidades del Estado y la sociedad en el desarrollo de un nuevo tipo de especialista capaz de pensar creativamente fuera de la caja. La formación de su potencial intelectual y competencia profesional se está convirtiendo en una tarea primordial para las instituciones educativas profesionales. Los procesos cognitivos, que incluyen creatividad, inteligencia y atención, actúan como base psicológica para la capacidad de los estudiantes de implementar soluciones innovadoras. El propósito del artículo es considerar la experiencia de la formación de la capacidad de los estudiantes para la actividad cognitiva como una de las actividades punteras en el desarrollo de la competencia de los estudiantes. Los procesos cognitivos son responsables del desempeño de la función cognitiva. La actividad cognitiva tiene como objetivo encontrar por parte del alumno soluciones a las tareas. En el proceso de esta actividad mental, el estudiante llega a comprender la esencia del problema y su solución. El estudio presenta la compleja dinámica de la actividad cognitiva, lo que propicia la aparición de soluciones innovadoras no estándar. Los resultados del estudio muestran un aumento en el nivel de actividad cognitiva de los estudiantes luego de la implementación de métodos, medios y tecnologías modernos en la formación de los estudiantes.

Palabras clave: educación a distancia, pandemia de coronavirus, educación profesional, interacción virtual.

\section{Summary}

The profound transformations that have taken place in the field of professional training of students have become a response to socio-economic changes in the country. Innovative processes are aimed at meeting the needs of the state and society in the development of a new type of specialist capable of creative thinking outside the box. The formation of their intellectual potential and professional competence is becoming a paramount task for professional educational institutions. Cognitive processes, which include creativity, intelligence and attention, act as a psychological basis for students' ability to implement innovative solutions. The purpose of the article is to consider the experience of the formation of students 'ability to cognitive activity as one of the leading activities in the development of students' competence. Cognitive processes are responsible for the performance of the cognitive function. Cognitive activity is aimed at finding the student solutions to the tasks. In the process of this mental activity, the student comes to an understanding of the essence of the problem and its solution. The study presents the complex dynamics of cognitive activity, prompting the emergence of non-standard innovative solutions. The results of the study show an increase in the level of cognitive activity of students after the implementation of modern methods, means and technologies in training students.

Key Words: distance learning, coronavirus pandemic, professional education, virtual interaction.

\section{Introducción}

Las transformaciones socioeconómicas activas han llevado al surgimiento de la necesidad que el Estado y la sociedad preparen un nuevo tipo de graduados (Filchenkova, 2019). La formación de un especialista competente moderno implica una intensa actividad cognitiva (Orlova and Lukashik, 2018). La actividad cognitiva se entiende como una actividad que conduce a cierto conocimiento, al establecimiento de los significados del estado actual de las cosas en el mundo (Tarnaeva and Osipova, 2016).

La necesidad de formar la capacidad para realizar la actividad cognitiva de los estudiantes está consolidada por el estándar educativo estatal federal (Klinkov, 2018). La formación de la 
capacidad de los estudiantes para la actividad cognitiva es una de las competencias clave en la preparación de los licenciados modernos (Donetskova, 2019).

La actividad cognitiva contribuye al desarrollo de la creatividad de los estudiantes, lo que, a su vez, permite que los estudiantes sean más libres para realizar las tareas asignadas, para elegir otras formas atípicas de resolverlas. Los estudiantes que son capaces de una actividad cognitiva activa son capaces de generar ideas innovadoras, que traducen en actividades de investigación.

Para el desarrollo de la actividad cognitiva, las instituciones de educación superior modernas buscan diversas formas innovadoras que les permitan involucrar a los estudiantes en actividades educativas activas (Chil- Núñez et al, 2020).

Un análisis teórico de los trabajos disponibles sobre el tema de los procesos cognitivos sugiere que algunos temas están insuficientemente desarrollados. La investigación adicional ayudará a llenar los vacíos existentes (Lutfullaev et al., 2020). (Chertovskikh, 2019).

\section{Marco teórico}

Entre los diversos tipos de actividades educativas de los estudiantes, es la cognitiva la que juega un papel significativo, ya que está directamente relacionada con los procesos de memoria, la capacidad de los estudiantes para dominar la información. La actividad implica el desarrollo de conocimientos, habilidades por parte de los estudiantes en el proceso de aprendizaje.

La actividad cognitiva está orientada a la formación del conocimiento (ArbeláezCampillo et al., 2020). En el trabajo de V.N. La actividad cognitiva de Druzhinin se presenta como un sistema cerrado, cuyos componentes son la adquisición y transformación del conocimiento, su preservación y aplicación (Druzhinin, 2007).

La capacidad de adquirir conocimientos, L.P. Tarnaeva y E.S. Osipova, es la capacidad de aprendizaje, la capacidad del estudiante para transformar el conocimiento se llama creatividad, la capacidad de aplicar los conocimientos adquiridos en condiciones reales se llama inteligencia (Tarnaeva and Osipova, 2016). El aprendizaje como componente de la actividad cognitiva es, en primer lugar, la capacidad de asimilar nuevos conocimientos y formas de actuar y, en segundo lugar, una medida de la eficacia de la actividad intelectual. La creatividad actúa como creatividad. ED Telegina identificó varias etapas principales de resolución de problemas: análisis de condiciones, desarrollo de un plan, desarrollo de hipótesis, su verificación (Akhmetshin, 2020), análisis de las condiciones iniciales y selección de la solución final (Eliseeva et al., 2020). La actividad cognitiva en la estructura de la competencia profesional permite a los estudiantes dominar nuevas formas de autorrealización profesional (Gutsu, 2013).

El proceso de desarrollo de la actividad cognitiva se lleva a cabo en etapas, por lo que se ajusta fácilmente y aumenta el potencial de aprendizaje (Pinkovetskaia et al., 2020). Las habilidades cognitivas se pueden mejorar continuamente, lo que es especialmente relevante en un entorno educativo en constante cambio (Pichugina and Bondarchuk, 2019). En el contexto del desarrollo de la actividad cognitiva, es necesario cumplir con los siguientes requisitos: la actitud del docente hacia el alumno como materia preparada para el autodesarrollo y el autoconocimiento (Ivanov et al., 2020); la formación de condiciones en las que el alumno podrá realizar sus habilidades (introspección, motivación, reflexión) (Birzhenyuk and Efimova, 2020); desarrollo de la trayectoria educativa individual de los estudiantes (Tsarapkina et al., 2019a); la formación de la competencia profesional (Garnevska, 2019). 


\section{Metodología}

Se realizó un estudio de los niveles de desarrollo de la actividad cognitiva de los estudiantes (alto, medio, bajo). Para ello, se identificaron los siguientes criterios: la integración de conocimientos profesionales; la necesidad de superación profesional, la adquisición de nuevos conocimientos profesionales; capacidad de ser creativo; capacidad para analizar situaciones profesionales; la capacidad de realizar reflexiones; flexibilidad y pensamiento crítico.

El nivel de desarrollo del criterio "integración del conocimiento profesional" refleja la coherencia y versatilidad del conocimiento en una disciplina particular, ideas sobre los patrones de desarrollo profesional. El criterio "la necesidad de mejorar los conocimientos profesionales" refleja no solo la necesidad de mejorar los conocimientos, sino también la capacidad de los estudiantes para hacerlo. La superación profesional es una parte integral de la actividad. Los estudiantes planifican sus programas de autodesarrollo. "Habilidad para ser creativo" muestra la capacidad para realizar tareas de una manera no estándar, el uso de habilidades creativas por parte del estudiante. "La capacidad de analizar situaciones profesionales" muestra la capacidad de los estudiantes para adaptarse a las condiciones profesionales en constante cambio, la búsqueda constante de nuevas soluciones. El criterio "Flexibilidad y criticidad del pensamiento" refleja la capacidad del alumno para cuestionar hechos y llevar a cabo una verificación adecuada de la información, elegir soluciones alternativas.

La muestra estuvo formada por 194 estudiantes. La población estudiada está representada por estudiantes de varias instituciones de educación superior. El tamaño de la muestra y los criterios de selección garantizan que la muestra sea representativa.

\section{Resultados y discusión}

En el contexto de la introducción de tecnologías innovadoras en el proceso educativo, la naturaleza de la actividad cognitiva cambia, se vuelve más diversa y rica. En las clases prácticas, los estudiantes practican las habilidades necesarias para la percepción adecuada y el procesamiento oportuno de la información educativa, por ejemplo, determinando la relevancia del material resaltando juicios iniciales e inferencias lógicas, detectando juicios infundados, elaborando resúmenes (Petrichev et al., 2018). Los estudiantes participan en discusiones, comentan las actuaciones de sus compañeros (Tishchenko, 2020). El docente no solo establece tareas y evalúa los resultados del trabajo, sino que también forma la capacidad de los estudiantes para la autoestima, para el autocontrol involucrándolo en una discusión abierta del problema (Malushko and Lizunkov, 2020).

En el proceso de formación de la capacidad de los estudiantes para la actividad cognitiva se involucran diversos medios, enseñanzas que aumentan la visibilidad (videos, diagramas, tablas, diagramas, fotografías, dibujos y otros); hardware (pizarrones inteligentes, cámaras de documentos, proyectores, computadoras y otros); equipo de demostración; plataformas educativas electrónicas; fuentes literarias ubicadas tanto en papel como en soportes electrónicos (libros de texto, diccionarios, libros de referencia, libros) (Kalinkina et al., 2017).

La peculiaridad de la formación de la capacidad para la actividad cognitiva es la organización del proceso cognitivo, la presentación del material. El docente desempeña el rol de consultor en el proceso educativo, sin embargo, estructura la lección educativa para que los estudiantes se sometan a un control oportuno, luego de lo cual pueden corregir sus actividades (Andrienko, 2019b). Para cada alumno se desarrollan varias tareas para procesar repetidamente 
diversas fuentes de información (Andrienko, 2019a). Como resultado, se logra una comprensión más profunda de esta información.

La interacción de los alumnos se lleva a cabo tanto en condiciones de aula como en formato remoto mediante el uso de tecnologías electrónicas. La actividad cognitiva se lleva a cabo utilizando la implementación de las capacidades de las plataformas educativas electrónicas y varios sistemas para la realización de clases a distancia.

Las tecnologías de diseño se utilizan en la organización de la actividad cognitiva de los estudiantes. Al completar proyectos individuales y grupales sobre diversos temas, los estudiantes desarrollan la capacidad de resolver de forma independiente y creativa los problemas que surgen en el proceso de realización del trabajo. Para desarrollar la capacidad de pensamiento crítico, juego, discusión, se implementan tecnologías de la información.

Se realizó un estudio de los niveles de desarrollo de la actividad cognitiva de los estudiantes (alto, medio, bajo). Para ello, se identificaron los siguientes criterios: la integración de conocimientos profesionales; la necesidad de superación profesional, la adquisición de nuevos conocimientos profesionales; capacidad de ser creativo; capacidad para analizar situaciones profesionales; la capacidad de realizar reflexiones; flexibilidad y pensamiento crítico.

El estudio involucró a 194 estudiantes de varias instituciones de educación superior.

La Tabla 1 presenta los resultados del procesamiento estadístico de datos para identificar el nivel de desarrollo de la actividad cognitiva de los estudiantes.

\begin{tabular}{|c|c|c|c|c|c|c|}
\hline \multirow[t]{3}{*}{ Criterio } & \multicolumn{3}{|c|}{ Año 2019} & \multicolumn{3}{|c|}{ Año 2020} \\
\hline & \multicolumn{6}{|c|}{ Niveles } \\
\hline & Alta & Media & Baja & Alta & Media & Baja \\
\hline Integración de conocimientos profesionales & $35 \%$ & $40 \%$ & $25 \%$ & $46 \%$ & $35 \%$ & $19 \%$ \\
\hline $\begin{array}{l}\text { La necesidad de mejora profesional y la } \\
\text { adquisición de nuevos conocimientos } \\
\text { profesionales. }\end{array}$ & $32 \%$ & $43 \%$ & $25 \%$ & $47 \%$ & $38 \%$ & $15 \%$ \\
\hline Instalación de creatividad & $39 \%$ & $40 \%$ & $21 \%$ & $45 \%$ & $41 \%$ & $14 \%$ \\
\hline $\begin{array}{c}\text { Capacidad para analizar situaciones } \\
\text { profesionales }\end{array}$ & $35 \%$ & $50 \%$ & $15 \%$ & $41 \%$ & $52 \%$ & $7 \%$ \\
\hline Capacidad de reflexión & $60 \%$ & $30 \%$ & $10 \%$ & $65 \%$ & $25 \%$ & $10 \%$ \\
\hline La flexibilidad y la criticidad del pensami & $52 \%$ & $38 \%$ & $10 \%$ & $54 \%$ & $35 \%$ & $13 \%$ \\
\hline
\end{tabular}

Tabla 1. Resultados del procesamiento estadístico de datos para identificar el nivel de desarrollo de la actividad cognitiva de los estudiantes

Se observó un alto nivel de integración del conocimiento profesional en 2019 en el 35\% de los estudiantes, en 2020 esta cifra aumentó al 46\%. "La necesidad de superación profesional, la adquisición de nuevos conocimientos profesionales" aumentó al 47\%. "La capacidad de crear" ha aumentado del 39\% al 45\%. "La capacidad de analizar situaciones profesionales" aumentó al 41\%. El 65\% de los estudiantes en 2020 tiene un alto nivel de capacidad para realizar la reflexión. Un alto nivel de flexibilidad y pensamiento crítico en 2020 tiene el 54\% de los participantes de la investigación. 
También se determinó el indicador de nivel medio.

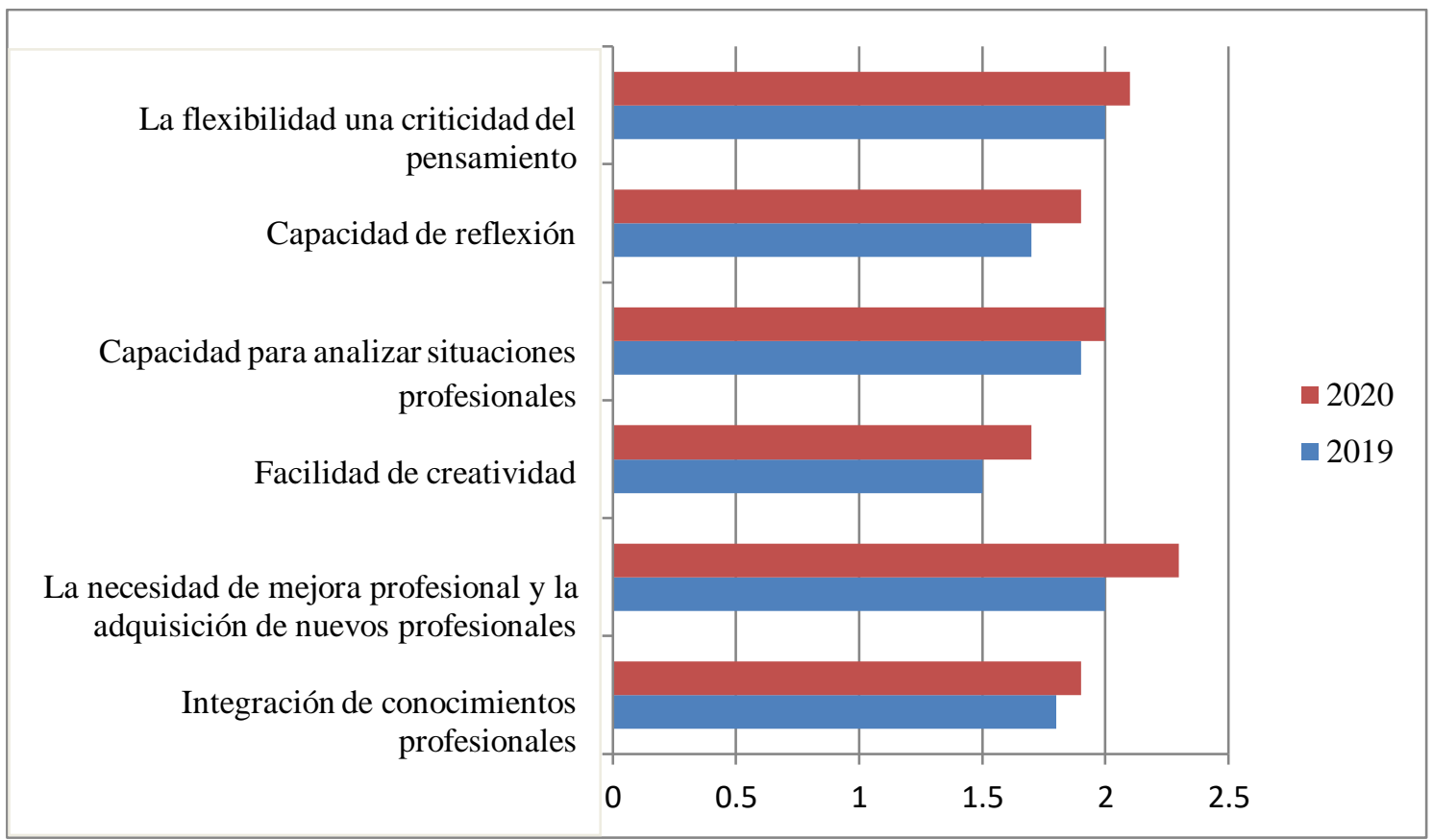

Figura 1. Resultados del procesamiento estadístico de datos para identificar el nivel de desarrollo de la actividad cognitiva de los estudiantes

Los indicadores de 2020 nos permiten hablar de la mejora en los resultados de los estudiantes. El promedio de niveles para 2020 es más alto.

\section{Conclusiones}

La organización del proceso educativo utilizando métodos, herramientas y tecnologías modernas puede mejorar la calidad de la formación de los estudiantes y aumentar el nivel de actividad cognitiva (Davydova, 2020). El estudio muestra que los indicadores para 2020 superan los indicadores para 2019. El nivel de actividad cognitiva de los estudiantes con la introducción de tecnologías y medios innovadores ha aumentado significativamente. Los estudiantes se han vuelto más independientes, listos para soluciones de problemas no estándar. Se puede afirmar que la mayoría de los participantes de la investigación tienen un nivel medio y alto de desarrollo de la actividad cognitiva. Poseen un conocimiento profundo, están preparados para la superación profesional y una alta actividad creativa. Sin embargo, vale la pena señalar a los participantes con bajos niveles de rendimiento cognitivo. Al poseer conocimientos sistémicos en el campo de disciplinas específicas, solo tienen ideas fragmentarias sobre el problema del desarrollo profesional y están listos para métodos de acción estereotipados, puede reflexionar.

Este estudio no agota todos los aspectos del problema planteado. En el futuro, señalamos la necesidad de reducir el número de estudiantes con un bajo nivel de actividad cognitiva y profundizar en el tema del desarrollo de la actividad cognitiva de los estudiantes en las condiciones modernas.

\section{Bibliographic references}

Akhmetshin, A.A. (2020). "Features of the activities of institutions of additional professional education in the context of the coronavirus pandemic in Russia". Innovation and Investment, No. 8, pp. 239-232. 
Andrienko, O.A. (2019a). "On the need to apply gaming training technologies". Balkan Scientific Review. Vol. 2, No 4, pp. 5-8.

Andrienko, O.A. (2019b). "Modern educational technologies: technology of self-presentation". Balkan Scientific Review. Vol. 1 No. 3, pp. 5-7.

Arbeláez-Campillo, D.F., Villasmil Espinoza, J. J. (2020). Escenarios prospectivos de un nuevo orden internacional que se vislumbra luego de la pandemia COVID-19." Revista Telos, No. 22 (3), pp. 494.

Arbeláez-Campillo, D., \& Rojas-Bahamon, M. (2020). Pandemics in globalization times. Amazonia Investiga, 9(27), 3-4. https://doi.org/10.34069/AI/2020.27.03.0

Birzhenyuk, G.M., \& Efimova, T.V. (2020). "Schering as a new landmark in education". Innovative development of vocational education. Vol. 2, No. 26, pp. 12-21.

Chertovskikh, O.O. (2019). "Prospects for the use of digital resources in education". Baltiyskiy gumanitarnyy zhurnal (Baltic Humanitarian Journal). Vol. 8, No. 4 (29), pp. 184-187. (in Russ.).

Chil-Núñez, I., Escalona Arranz, J., Molina Bertrán, S., Dutok Sánchez, C., Arias Ramos, D., Pérez Rondón, L., OchoA Pacheco, A., \& Picanço Souto, R. (2020). Perfeccionamiento de la estrategia curricular de medio ambiente de la carrera de ciencias farmacéuticas de la Universidad de Oriente, Cuba. Revista Científica Del Amazonas, 3(5), pp. 6-17. https://revistadelamazonas.info/index.php/amazonas/article/view/24

Davydova, T.E. (2020). "The specifics of the organization of the educational process at the university in non-standard conditions". Production organizer. Vol. 28, No. 2, pp. 97-107.

Donetskova, O. YU. (2019). "Modernization of the modern education system in Russia". In: Baltiyskiy gumanitarnyy zhurnal (Baltic Humanitarian Journal). Vol. 8, No. 2 (27), pp 37-39. (in Russ.).

Druzhinin, V.N. (2007). Psychology of general abilities: a tutorial / V.N. Druzhinin. - 3rd edition. - St. Petersburg: Peter, 368 p.

Eliseeva, D.YU., Fedosov, A.YU., Agaltsova, D.V., Mnatsakanyan, O.L., Kuchmezov, KH.KH. (2020). The evolution of artificial intelligence and the possibility of its application in cyber games. Amazonia Investiga. Vol. 9 No. 28, pp. 123-129.

Filchenkova, I.F. (2019). "Educational management of innovative activity of teachers as an object of pedagogical research". Vestnik Mininskogo universiteta (Vestnik of Minin University). Vol. 7, (4), 3. (in Russ.).

Gutsu, E.G. (2013). Cognitive component in the structure of the professional competence of a higher school teacher, Modern problems of science and education. 1.

Garnevska, S.M. (2019). Professional development of teachers - opportunities, norms, realization. Scientific Vector of the Balkans, 2(4), 13-17.

Ivanov, M.S., Parnikova, T.A., Gulyaev, V.P., \& Petrov, N.V. (2020). The activity approach implementation in the formation of students' general technical competencies. Amazonia Investiga. No. 9(26), pp. 205-210. 
Kalinkina, E.G., \& Gorodetskaya, N.I. (2017). "Development of e-learning and distance learning technologies in the process of professional development of teachers". Nizhny Novgorod education. No. 1, pp. 131-138.

Klinkov, G.T. (2018). "The specificity of manifestation of pedagogical communication as a special construct". Scientific Vector of the Balkans. No. 1, pp. 51-52.

Lutfullaev, G. A., Lutfullaev, U. L., Kobilova, S. S., \& Neymatov, U. S. (2020). Experience of distance learning in the context of the COVID-19 pandemic. Problems of pedagogy, No.4 (49), pp. 66-69.

Malushko, E. Y., \& Lizunkov, V. G. (2020). Electronic education system as a tool for increasing the competitiveness of a specialist in the conditions of digital economy. Vestnik Mininskogo universiteta (Vestnik of Minin University). 8 (2 (31)), 3. (in Russ)

Orlova, I.G., \& Lukashik, A.F. (2018). "Distance learning as a way to implement advanced training of specialists in the fuel and energy complex (on the example of FGAOU DPO "KEMRIPK")". Bulletin KEMRIPK. No. 2, pp. 46-49.

Petrichev, P. V., Masyuk, N. N., \& Bushueva, M. A. (2018). "Method of estimation of the effectiveness of the partnership russian universities with foreign educational organizations". Azimut nauchnykh issledovaniy (Azimuth of Scientific Researches: Economics and Management). Vol.7, No. 3 (24), pp. 229-232.

Pinkovetskaia, I., Arbeláez-Campillo, D., Rojas-Bahamón, M., \& Veas Iniesta, D. (2020). Motivation of new entrepreneurs in modern economies. Amazonia Investiga, 9(29), pp. 368-373. https://doi.org/10.34069/AI/2020.29.05.41

Pichugina, G.A., \& Bondarchuk, A.I. (2019). "Structure of the training case in the organization of the educational process". Humanitarian Balkan Research. Vol. 2, No. 4, pp. 5-7.

Tishchenko, A.S. (2020). "Assessment of the losses of educational organizations from a reduction in the volume of additional educational services to the population during a pandemic". Economic Development of Russia. Vol. 27, No. 6, pp. 73-79.

Tsarapkina, JU. M., Dunaeva, N. V., \& Kireicheva, A. M. (2019a.) "Application of BYOD technology in education on the example of Lecture Racing mobile application". Informatika i obrazovanie - Informatics and Education. No. 9 (308), pp. 56-64.

Tarnaeva, L. P., \& Osipova, E. S. (2016). Cognitive activity of students in the process of using the resources of corpus linguistics in the formation of the idiomatic competence of a translator in the field of professional communication. Philological sciences. Questions of theory and practice, (11-1 (65)), pp. 198-201. 